\title{
Characterization of Skin Lesion Texture in Diffuse Reflectance Spectroscopic Images *
}

\author{
Mehrübe Mehrübeoğlu, Nasser Kehtarnavaz \\ Department of Electrical Engineering \\ Texas A\&M University \\ College Station, Texas
}

\author{
Guillermo Marquez, Lihong V. Wang \\ Biomedical Engineering Program \\ Texas A\&M University \\ College Station, Texas
}

\begin{abstract}
This paper examines various texture features extracted from skin lesion images obtained by using diffuse reflectance spectroscopic imaging. Different image texture features have been applied to such images to separate precancerous from benign cases. These features are extracted based on co-occurrence matrix, wavelet decomposition, fractal signature, and granulometric approaches. The results so far indicate that fractal and wavelet-based features are effective in distinguishing precancerous from benign cases.
\end{abstract}

\section{Introduction}

New methods of non-invasive skin analysis have been an ongoing research. Multiple groups have worked on noninvasive methods for detecting abnormal cells [1]-[3]. According to the annual Cancer Statistics Review, an estimated 42,000 new cases of melanoma of the skin in the U.S. was reported for 1998 [4]. Visual inspection of the lesions by the doctor does not always result in conclusive decisions on the state of the lesion. The current methods of skin analysis involve removing a tissue sample from the body surface, and performing biopsy to determine whether the tissue is benign or precancerous. This invasive technique is unpleasant for the patient. It is also time consuming, since the biopsy report can take up to several weeks to be completed, and sometimes impossible, if the patient has too many lesion sites. The doctors could greatly benefit from a fast and reliable system which could enhance the separation of benign and precancerous skin lesions without the need for biopsy.

In this paper, a non-invasive optical method based on image processing techniques is reported to identify benign and

\footnotetext{
1* E-mail contact: kehtar@ee.tamu.edu This work is partly supported by a US Department of Education graduate assistantship.
}

precancerous tissues. This developed diffuse reflectance spectroscopic imaging method does not require the removal of skin samples. Data acquisition involves shining white light onto the skin surface obliquely, and detecting the diffusely reflected light by a set of fibers. Each fiber provides a 1-D signal, which can then be combined to produce an image, increasing the extractable amount of information. The following sections describe the image acquisition and processing techniques, and the feature extraction methods used in separating two types of tissues, namely, benign and precancerous.

\section{Image acquisition}

Image acquisition was performed at UT MD Anderson Cancer Center in Houston with the help of an experienced dermatologist. White light (Oriel, 75 W Xenon Arc Lamp) was coupled to a fiber optic probe made from brass tubing, and $200 \mu \mathrm{m}$ diameter, low-OH optical fibers. The source fiber was oriented at a $45^{\circ}$ angle of incidence. Thirteen normally incident collection fibers, arranged in a linear array and spanning $0.35 \mathrm{~cm}$, collected the diffusely reflected light (Fig. 1).

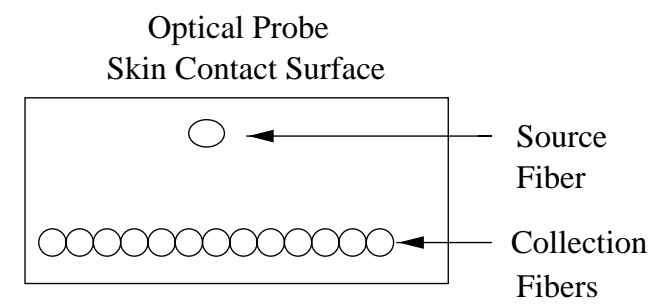

Figure 1. Optical probe for light transmission. The source fiber transmits the white light onto the skin surface. The diffusely reflected light off the skin surface is collected by a series of thirteen optical collection fibers. 
The outputs from these collection fibers were then placed at the object plane of the imaging spectrograph (Oriel, Multispec 257). The spectrograph spectrally dispersed the 1$\mathrm{D}$ light distribution and projected the image onto the CCD matrix (Princeton Instrument Inc., 1530P) as in Fig. 2. The vertical dimension of the image represented the spatial distribution of the diffuse reflectance. The horizontal dimension represented the spectral distribution for light from each collection fiber. A personal computer was used to automatically record the spectra of the collected white light.

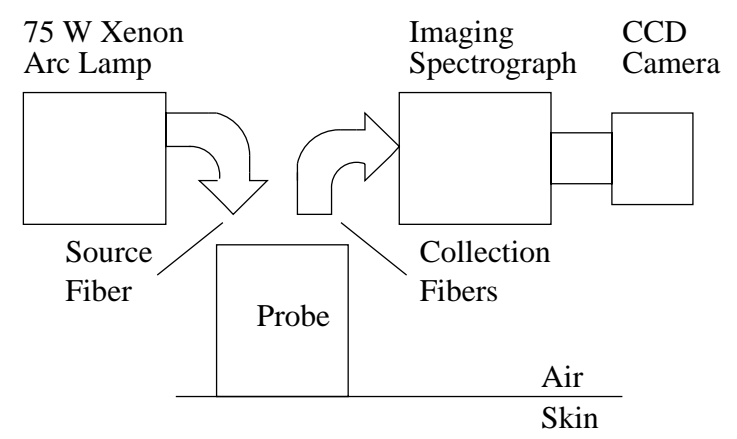

Figure 2. Diffuse Reflectance Spectroscopic Imaging System. The spectral information from the diffusely reflected light off the skin surface are transmitted to the CCD camera system via the probe and the imaging spectrograph.

A sample diffuse reflectance spectral image is shown in Fig. 3. The spectral intensity of the same image is presented in Fig. 4.

Spectral images were acquired from 18 skin lesion cases. The biopsy reports identified 8 of the lesions as benign, and the remaining 10 as precancerous. For each case, 3 images were obtained from the lesion site, and 3 images from the neighboring healthy tissue, with the exception of the first case, where 5 images were collected each for lesion and healthy sites, for a total of 112 image samples. In each image acquisition, the probe was removed and repositioned to obtain the images from different locations on the lesion and the healthy skin. Before each image was stored, background subtraction was executed to remove dark room camera system noise. The resulting image was then calibrated against the k-factor of each fiber, since each fiber had a different transmission/absorption curve.

\section{Image texture features}

The major task of this work involved the study of image texture features to identify effective texture features in the spectral skin images. A wide collection of image texture features based on co-occurrence matrix, wavelet decomposition, fractals, granulometries were extracted and examined to obtain those features whose distributions were relatively non-overlapping for benign and precancerous cases.

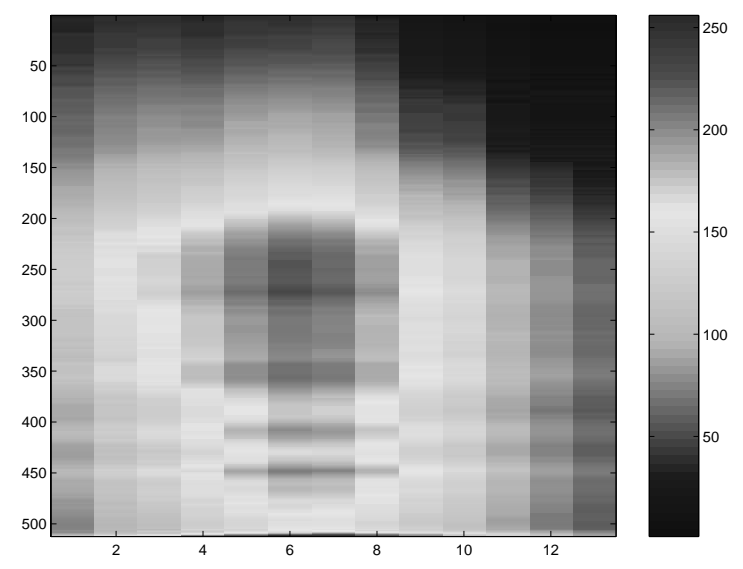

Figure 3. A sample image. The horizontal axis represents the location of fibers. The vertical axis reflects the wavelength at which the light was collected. Different shades of gray in the image represent the relative intensity of the received light.

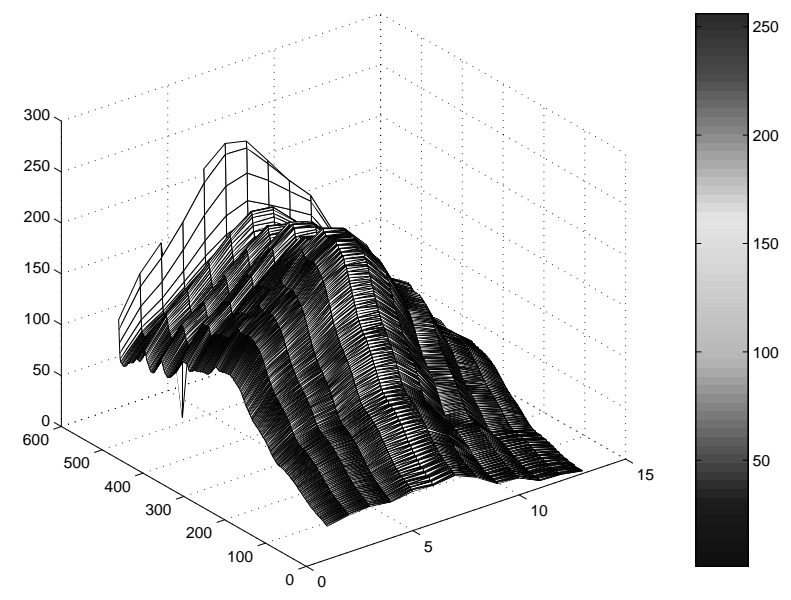

Figure 4. The spectral intensity of the image in Fig. 2. The vertical axis represents the image intensity at different fiber locations and wavelengths.

Unfortunately there is no universally agreed upon definition of texture in the image processing literature. That is why many different mathematical approaches have been used to define texture features. Basically, the term texture is 
used to reflect variations in a statistical sense in gray level values. Image texture analysis has been applied to various types of medical images to identify different types of tissues, in particular benign versus malignant. There are no successful classification of benign and precancerous skin tissues in the reviewed literature. An overview of the texture features examined in this study is provided below.

\subsection{Co-occurrence matrix texture features}

Co-occurrence matrix statistics introduced by Haralick [5],[6] constitute the most widely used image texture features. Experiments involving human visual system have revealed that second order statistics play a major role in visual description of texture. Co-occurrence matrix is a realization of this attribute.

A first order histogram shows the gray level distribution within an image. However, it does not provide any information about the relationship of gray levels in the image. A second order histogram based on the co-occurrence matrix, on the other hand, shows such in formation by considering pixel pairs. For an 8-bit image having 256 gray levels, the second order histogram represents all possible gray level pairs as a 256x256 matrix. Each entry in this matrix denotes the number of occurrences of a pair of gray level values being a distance $d$ apart at an orientation angle $\theta$. Normally four orientations corresponding to $0^{\circ}$ (horizontal), $45^{\circ}$ (diagonal), $90^{\circ}$ (vertical), and $135^{\circ}$ (anti-diagonal) are used.

Based on the co-occurrence matrix, the following texture features are defined: standard deviation, correlation, maximum probability, contrast, inhomogeneity, inverse difference moment, and entropy. In this study, these features were examined.

\subsection{Wavelet-based texture features}

Multi resolution wavelet analysis is a recently developed scheme [7] that represents data at various scales with different time or spatial resolutions. The concept of wavelet analysis is similar to the scale used in maps. High scales correspond to non-detailed global views of a map, while low scales to small, detailed or closer views. Thus low scales are closely related to the notion of high frequency that last for a very short time or spatial duration. At high scales (corresponding to low frequency in Fourier sense), the time or spatial resolution is high, while at low scales it is low. The aim is to capture high frequency content in a signal or an image as more often than not, the high frequency contents (edges) occur within a short time or spatial resolution.

Wavelet decomposition of images takes place in a similar manner. Instead of time, two spatial dimensions $\mathbf{x}$ (rows) and $\mathbf{y}$ (columns) are involved. The two-dimensional discrete wavelet transform of an image is separable. This al- lows the discrete wavelet transform to be first applied in one-dimension across the rows of the image. Each row (treated as a one-dimensional signal) is then decomposed into two equal half-length coarse and detail components. The resulting transformed rows are placed in an intermediate matrix. The discrete wavelet transform is again applied to the columns of the intermediate matrix.

Such a decomposition yields a transformed image with the same height and width as the original image. As a result, four subimages are generated, each half in resolution with respect to the height and width. Thus a $256 \times 256$ image is decomposed into four $128 \times 128$ subimages. These subimages comprise one coarse and three detail subimages. The horizontal (H), diagonal (D), vertical (V) component subimages correspond to the high frequency, while the coarse component subimage (C) correspond to the low frequency part of the image. The next level of decomposition is done on the coarse subimage generating four other subimages similar to the first level of decomposition.

Two texture features are then derived from each subimage. These features are called energy and entropy. In this study, various types of wavelets were investigated, in particular Daubecheis and spline wavelets.

\subsection{Fractal signature texture features}

Fractal geometry has been employed to define texture features [8]. A fractal is a set for which the so-called Hausdorff Dimension is strictly greater than the topological dimension, the intuitive notion of dimension. A signal or image can be considered to be a fractal having a shape composed of parts similar to whole in some way. The concept of self-similarity at all scales is specified by a measure known as Fractal Dimension (FD) (simplified form of Hausdorff Dimension). This dimension is different from the topological dimension. For example, we normally consider curves having a dimension of 1 , surfaces having a dimension of 2 , and solids having a dimension of 3 , while fractal dimension characterizes their roughness.

There exist a number of approaches to compute fractal dimension from images. Among them, multi-resolution fractal analysis evaluates fractal dimensions at different scales, thereby taking into account possible variations in modeling image texture as fractal surfaces. Fractal dimension obtained in this manner is known as fractal Signature $(S)$. In order to model texture as fractals, fractal signature $S(\varepsilon)$ for each successive scale $\varepsilon$ is computed from three consecutive points $(\log (\varepsilon-1), \log (A(\varepsilon-1)))$, $(\log (\varepsilon), \log (A(\varepsilon))),(\log (\varepsilon), \log (A(\varepsilon+1)))$ using the linear regression technique, where $A(\varepsilon)$ denotes the image surface area at scale $\varepsilon$. Fractal signature $S(\varepsilon)$ is the slope of the best-fit line through these points. In this study, many (20) scales for 4 as well as 8-neighborhood points were used to 
compute fractal texture signatures or features.

\subsection{Granulomteric texture features}

Granulometric texture features are based on morphological granulometric size distributions describing the granularity within a binary image. Moments of size distributions serve as granulometric features. The basic idea is to characterize a binary image as a collection of grains and then sieve the grains. As the mesh size of the sieve is increased gradually, the residual area of the sieved image gets reduced since only larger grains fall through the increasing size sieve. Thus a decreasing size distribution of the residual area is obtained that serves as an indicator of texture of the binary image. Sieving is done by a morphological operation called opening, and the sequence of sieved images is called a granulometry. Opening operation sieves an image by only passing those parts that contain a specific shape known as a structuring element.

The above granulometric approach has been extended from binary images to gray level images [9]. Similar to the binary case, a series of openings by a sequence of expanding structuring elements results in reduced gray level heights of an image surface. This generates a decreasing sequence $\Omega(k)$ of the volumes underneath the opened images, where $k$ denotes the opening index. The normalization $\Phi(k)=1-\Omega(k) / \Omega(0)$ is considered to be a probability distribution function and the moments of the corresponding density function $d \Phi(k) / d(k)$, named pattern spectrum, are taken to be granulometric texture features. Normally the first three moments mean, variance, and skewness are used.

Different structuring elements can be employed to probe an image surface. In this study, structuring elements considered included flat linear along directions $45^{\circ}$ and $-45^{\circ}$, flat top and cone shapes.

\section{Feature analysis}

Before performing texture feature analysis, the image intensity was mapped between 0 and 255 for each individual image for the 8-bit texture feature algorithms. Then the above-mentioned texture features were extracted to examine the separability of distributions between benign and precancerous tissue images: Tested texture features were derived from co-occurrence matrix, wavelet, fractal signatures, and granulometry approaches, as explained above [10].

The effective features were determined by calculating the Fisher discriminant or distance for each feature set. Fisher distance is a measure of class separability [11]. The mean and standard deviation of the feature values in each class are used to compute the Fisher distance. For a two- class problem as in this study, the Fisher distance is given by

$$
F_{b p}=\left(\mu_{b}-\mu_{p}\right)^{2} /\left(\sigma_{b}^{2}+\sigma_{p}^{2}\right)
$$

where $\mu_{b}$ is the mean of the feature values for the benign class, $\mu_{p}$ is the mean of the feature values for the precancerous class, $\sigma_{b}$ is the standard deviation of the feature values for the benign class, and $\sigma_{p}$ is the standard deviation of the feature values for the precancerous class.

As can be seen from the above equation, the Fisher distance increases as the difference between the means of the feature values for the two classes increases, or as the separability between the two classes increases. In a similar manner, as the standard deviation of the feature values in each class decreases, the Fisher distance increases. This indicates that the extracted features can be ranked using the Fisher distance, and features that generate relatively high Fisher distances can be considered effective.

\section{Table 1. Typical Fisher distances used to de- cide feature selection.}

\begin{tabular}{|l|l|}
\hline Features & Fisher Distance \\
\hline fractal (4 neighborhood) & 1.8729 \\
wavelet (Daubecheis energy) & 0.6995 \\
co-occurrence (standard deviation) & 0.1135 \\
granulometry $\left(45^{\circ}\right.$ cone) & 0.0148 \\
\hline
\end{tabular}

Figures 5 and 6 show sample plots of the texture features, namely the fractal and wavelet based features, that were found to be effective in separating precancerous from benign cases.

In these figures, feature values from a lesion image were calibrated against feature values from the corresponding healthy images by performing feature division. The benign and precancerous labels were determined by biopsy. As can be seen, each of these features generated one false negative. By using both of these features simultaneously as the input to a classifier, it is possible to avoid such false cases. For the database studied, the results indicate that the fractal and wavelet texture features are effective in separating precancerous from benign cases.

\section{Conclusions and future work}

Image processing texture feature extraction provides a non-invasive method in separating benign from precancerous skin lesions. Finding effective texture features that de- 


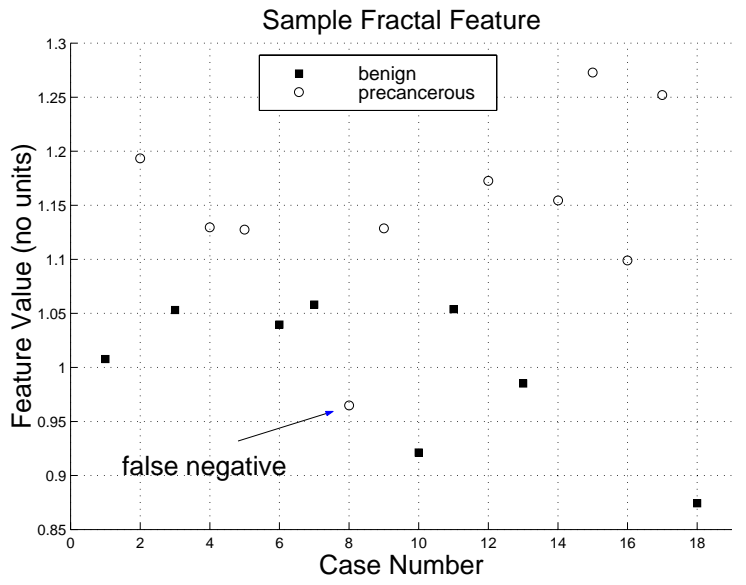

Figure 5. Sample plot of fractal texture feature for benign and precancerous tissue images. The horizontal axis represents the image index or case number, and the vertical axis represents the feature value.

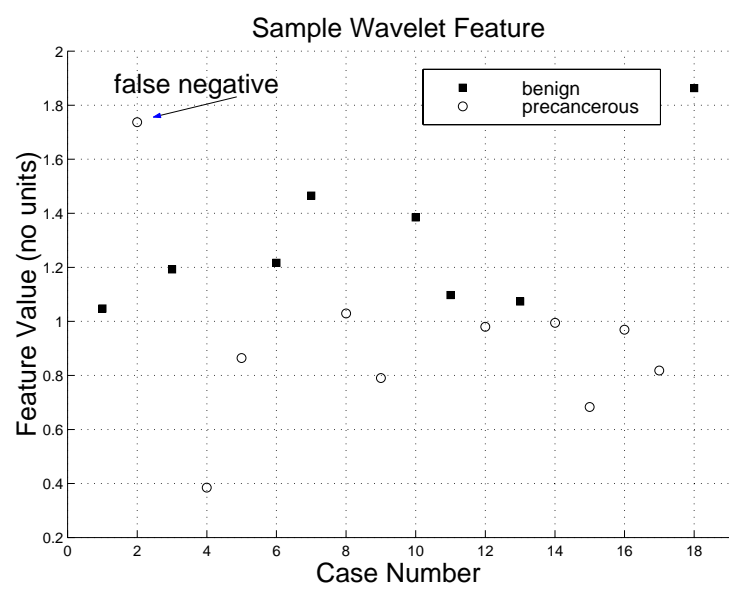

Figure 6. Sample plot of wavelet-based texture feature for benign and precancerous tissue images. The horizontal axis represents the image index or case number, and the vertical axis represents the feature value, as in Figure 5.

scribe each class most distinctly is the most challenging task in this image classification problem. In this work, various texture features were extracted from benign and precancerous skin lesion images. Results so far show that skin lesions can be characterized using appropriate image texture features. This is a promising finding for the future of noninvasive skin lesion classification. More images are to be acquired and added to the current database to increase the confidence of this finding. In addition, the features found effective will be used as the input to a statistical or neural net classifier for carrying out a ROC analysis.

\section{References}

[1] Advances in Optical Imaging and Photon Migration, Technical Digest, Optical Society of America, Washington, D.C., 1998.

[2] K. Tumer, N. Ramanujam, J. Ghosh, and R. RichardsKortum, "Ensembles of Radial Basis Function Networks for Spectroscopic Detection of Cervical Precancer," IEEE Transactions on Biomedical Engineering, vol. 45, no. 8, 1998, pp. 953-961.

[3] G. Marquez, and L.-H. Wang, "White light oblique incidence reflectometer for measuring absorption and reduced scattering spectra of tissue-like turbid media ," Optics Express, vol. 1, 1997, pp. 454-460. (Available at http://epubs.osa.org/opticsexpress/)

[4] Statistical Abstract of the United States 1998, U.S. Census Bureau, Washington, D.C., p. 152, Table 239, 1998.

[5] R. Haralick, Computer and Robot Vision, AddisonWesley, Massachusetts, 1992.

[6] C. H. Chen, L. F. Pau, and P. S. P. Wang, Handbook of Pattern Recognition and Computer Vision, World Scientific, New Jersey, 1998.

[7] S. Theodoridis and K. Koutroumbas, Pattern Recognition, Academic Press, San Diego, CA, 1999.

[8] M. J. Turner, J. M. Blackledge, and P. R. Andrews, Fractal Geometry in Digital Imaging, Academic Press, London, 1998.

[9] Y. Chen and E. R. Dougherty, "Gray-scale morphological granulometric texture classification," Optical Engineering, vol. 33, no. 8, 1994, pp. 2713-2722.

[10] F. Hussain, "Image Texture Analysis of Elastograms," M.S. Thesis, Department of Electrical Engineering, Texas A\&M University, December 1999, pp. 1-60.

[11] R. A. Vaughan, Pattern Recognition and Image Processing in Physiscs, (Proceedings of the Thirty-Seventh Scottish Universities Summer School in Physics, Dundee, August 1990), Adam Hilger, Bristol, 1990, pp. 250-251. 